\title{
Todesfälle in neun Regionen Italiens im Februar/März 2020: „Mortalitäts-Exzess-Lupe“ für SARS-CoV-2/COVID-19- Epidemiologie in Deutschland
}

\section{Deaths in nine regions of Italy in February/March 2020: "Mortality Excess Loupe" for SARS-CoV-2|COVID-19-Epidemiology in Germany}

\author{
Autoren \\ Peter Morfeld, Thomas C Erren
}

Institut

Institut und Poliklinik für Arbeitsmedizin, Umweltmedizin und Präventionsforschung, Uniklinik Köln, Köln

\section{Schlüsselwörter}

SARS-CoV-2/COVID-19, SMR, Mortalitäts-Exzess-Lupe, Epidemiologie, Surveillance

\section{Keywords}

SARS-CoV-2/COVID-19, SMR, mortality excess loupe, epidemiology, surveillance

\section{Bibliografie}

DOI https://doi.org/10.1055/a-1160-5859

Online-Publikation: 30.4.2020

Gesundheitswesen 2020; 82: 400-406

(c) Georg Thieme Verlag KG Stuttgart · New York ISSN 0941-3790

\author{
Korrespondenzadresse \\ Dr. Peter Morfeld \\ Institut für Arbeitsmedizin, Umweltmedizin und \\ Präventionsforschung \\ Uniklinik Köln \\ Kerpenerstraße 62 \\ 50937 Köln \\ peter.morfeld@rub.de
}

\section{ZUSAMMENFASSUNG}

Italien ist besonders durch SARS-CoV-2/COVID-19 betroffen. Kürzlich haben Colombo und Impicciatore die Todesfallzahlen in 1084 ausgewählten Gemeinden im Zeitfenster 21.2.2020 bis zum 21.3.2020 mit den entsprechenden Todesfallzahlen in den gleichen Zeitfenstern 2015 bis 2019 verglichen. Wir erweitern Auswertungen von Daten des Nationalen Instituts für Statistik Italiens (ISTAT) und berechnen Standardisierte Mortalitäts-Ratios (SMRs) in den neun ausgewählten Regionen Italiens, getrennt für Männer und Frauen und zusammengefasst. Wir analysieren Kovariableneinflüsse mit Poisson-Modellen und diskutieren Limitierungen dieser aktuellen Ausarbeitungen. Wir schlussfolgern: In Übereinstimmung mit Colombo und Impicciatore kann in der besonderen Corona-Situation diese „Mortalitäts-Exzess-Lupe“ - bei Annahme sonst konstanter Determinanten für Todesfälle - ein Virus-Test-unabhängiges Instrument sein, Mortalitätseffekte von SARS-CoV-2 zu bestimmen. Die aktuelle „Lupe“ ist fokussiert auf Gemeinden mit Zunahmen um mehr als 20\% an Todesfällen im März 2020 im Vergleich zu den durchschnittlichen Todesfällen an denselben Tagen in den Jahren 2015-2019. Man könnte das Zeitfenster der Untersuchung vor dem 21.2.2020 öffnen, um maskierte Mortalitätszunahmen bereits vor der ersten Meldung eines „COVID-19-Todesfalls“ festzustellen. Die aktuelle „Lupe“ vermittelt ausgeprägte Mortalitätsanstiege auch in Regionen, die nicht als Corona-Hotspot galten. Insofern können Mortalitätsdaten auch bei Fehlen repräsentativer Virus-Testergebnisse wichtige Hinweise auf die Verteilung oder Ausbreitung eines neu wirkenden Faktors sein. In der Gesamtschau ist es angezeigt, SMR-Analysen für Deutschland regelmäßig durchzuführen, differenziert nach Regionen, Geschlechtern, Altersgruppen und Todesursachen. Solche Analysen können zur Früherkennung und Beurteilung des Schweregrads einer tödlichen Pandemie („Krankheitslast“) sowie zur Überwachung der dynamischen Verbreitung eines Faktors wie SARS-CoV-2 beitragen. SMR-Analysen können auch genutzt werden, sowohl erwünschte als auch unerwünschte Auswirkungen von Maßnahmen gegen SARS-CoV-2/COVID-19 - und ggfs. andere Epidemien und Pandemien - abzuschätzen und zu evaluieren.

\section{ABSTRACT}

Italy is particularly affected by SARS-CoV-2/COVID-19. Recently, Colombo and Impicciatore compared the deaths in 1084 selected municipalities between 21 February 2020 and 21 March 2020 with deaths in the same time period in 2015 to 2019. We extend analyses of data from the Italian National Institute of Statistics (ISTAT) and calculate SMRs for all causes of death in the nine selected regions of Italy, separately for men and women and summarized. We analyze the effect of covariables by Poisson modelling and discuss the limitations of the current 
elaborations. We conclude: In agreement with Colombo and Impicciatore, in the particular corona situation, this "mortality excess loupe" - assuming otherwise constant determinants of death - can be a virus-test-independent tool to determine mortality effects of SARS-CoV-2. The current "loupe" is focused on municipalities with increases of more than $20 \%$ deaths in March 2020 compared to the average deaths on the same days in 2015-2019. The time window of investigation could be opened before 21 February 2020 to detect masked increases in mortality before the first "COVID-19 death" was ascertained. The current "loupe" conveys pronounced mortality increases also in regions that were not considered to be corona hotspots. In this respect, even in the absence of representative virus test results, mortality data can be important indicators of the distribution or spread of a newly acting factor. Overall, it is advisable to carry out SMR analyses for Germany on a regular basis, differentiated by region, gender, age group and cause of death. Such analyses can contribute to the early detection and evaluation of the severity of a deadly pandemic ("burden of disease") as well as to monitoring the dynamic spread of a factor such as SARS-CoV-2. SMR analyses can also be used to assess and evaluate both desired and undesired effects of measures taken against SARS-CoV-2/COVID-19 - and possibly other epidemics or pandemics.

\section{Einleitung}

Am 11. März 2020 erklärte die Weltgesundheitsorganisation den COVID-19-Ausbruch zu einer globalen Pandemie ${ }^{1}$. Italien ist besonders betroffen [1, 2]. Bisherige Darstellungen zur Bedeutung von COVID-19 für die Mortalität der infizierten Bevölkerung beschränkten sich - neben Modellhochrechnungen [3] - auf die Angabe absoluter Fallzahlen von Todesfällen mit positivem SARS-CoV-2-Test ${ }^{3}$. Diese Vorgehensweise erlaubt keinen Schluss auf eine ursächliche Beteiligung des Virus an all diesen Todesfällen (potentielle Überzuordnung). Andererseits kann diese Vorgehensweise nicht sicherstellen, dass alle betroffenen Todesfälle erfasst wurden (potentielle Untererfassung). Zudem ist ohne einen entsprechenden Vergleich mit der Mortalität in Vorjahren nicht klar, ob die beschriebene absolute Zahl der Todesfälle geeignet ist, eine Übersterblichkeit für das Frühjahr 2020 zu messen. Um eine bessere Orientierung zu geben, werden im Folgenden Standardisierte Mortalitäts-Ratios (SMRs) zur Gesamtmortalität berechnet und ausgewertet. Dies ist für ausgewählte Regionen und Gemeinden Italiens für den Zeitraum 21.02.2020 bis 21.03.2020 möglich.

\section{Methodik}

Unsere Auswertung stützt sich auf Colombo und Impicciatore 2020 [4], die Daten des Nationalen Instituts für Statistik Italiens (ISTAT) ${ }^{4}$ verwendeten [5]. Für diese Untersuchung wurden alle italienischen Gemeinden selektiert, die a) Daten vom 1. Januar bis 21. März 2020 an ISTAT geschickt haben, b) mindestens zehn Todesfälle vom 1. Januar 2020 bis 21. März 2020 aufwiesen und c) im März 2020 einen Anstieg der Todesfälle um mehr als $20 \%$ im Vergleich zum Durchschnittswert für den gleichen Zeitraum der Jahre 2015-2019 verzeichneten. Die Autoren wählten den 21. Februar 2020 als Start-

1 https://www.who.int/dg/speeches/detail/who-director-general-s-openingremarks-at-the-media-briefing-on-covid-19---11-march-2020

2 https://www.dgepi.de/assets/Stellungnahmen/Stellungnahme2020 Corona_DGEpi-21032020.pdf

3 Siehe die täglichen Darstellungen des Robert Koch-Instituts, Berlin: https://ww.rki.de/DE/Content/InfAZ/N/Neuartiges_Coronavirus/Fallzahlen. html und der Johns Hopkins University, Baltimore:

https://gisanddata.maps.arcgis.com/apps/opsdashboard/index.html\#/ bda7594740fd40299423467b48e9ecf6

4 https://www.istat.it/it/archivio/240401 punkt für das Auswertungs-Zeitfenster, denn am 21. Februar - so die Autoren - wurde der erste einheimische COVID-19-Todesfall gemeldet. Die „Mortalitäts-Exzess-Lupe“ wurde auf die Gemeinden in c) fokussiert. Die Untersuchung schloss Daten vom 21. Februar bis 21. März 2020 ein.

Wir berechnen aufgrund der Tabelle 1 in [4] SMRs für alle Todesursachen in den neun ausgewählten Regionen Italiens (beschränkt auf die selektierten Gemeinden), getrennt für Männer und Frauen und zusammengefasst: Beobachtet = Anzahl Todesfälle $2020 \mathrm{im}$ Zeitraum 21.2.-21.3.; Erwartet = Median der Anzahlen Todesfälle 2015-2019 im Zeitraum 21.2.-21.3; Mortalitätsratenverhältnis, nach Berücksichtigung von üblicherweise Alter und Geschlecht (indirekt standardisierte Mortalitäts-Ratio): SMR = Beobachtet/Erwartet mit $95 \%$-Konfidenzintervallen (95\%-KI) nach Poissonverteilung $[6,7]$.

Wir bestimmen Poisson-Modelle zur Schätzung und zufallskritischen Prüfung des Einflusses von Geschlecht und Regionen auf die SMR [6-8], auch mit Überdispersionsparameter (negative Binomialverteilung) [9].

Die Auswertung erfolgt mit Stata 14 [10].

\section{Ergebnisse}

Die Anzahl der selektierten Kommunen betrug 1084. Sie stammen aus neun Regionen Italiens (Piemonte e VdA, Lombardia, TrentinoA.A., Veneto, Friuli-V.G., Liguria, Emilia-Romagna, Centro, Sud e isole); die Größe der zugehörigen Quellpopulation wird mit 12,3 Mio. Einwohnern angegeben [4].

Die wesentlichen Ergebnisse unserer SMR-Analyse stellen wir in den $>$ Tab. 1-3 vor.

Alle SMRs liegen mit ihren unteren 95\%-Konfidenzintervallgrenzen oberhalb des Neutralwertes von 1. Für die neun Regionen gemeinsam beträgt die SMR der Gesamtmortalität 1,79 (95\%-KI: 1,76-1,81); die SMR für Männer liegt bei 1,96 (95\%-KI: 1,92-1,99) und für Frauen bei 1,64 (95\%-KI: 1,60-1,67).

Für die Lombardei werden folgende SMRs beobachtet: insgesamt 2,13 (95\%-KI: 2,09-2,17), für Männer 2,40 (95\%-KI: 2,342,47 ) und für Frauen 1,90 (95\%-KI: 1,85-1,95).

In einem Poisson-Modell, das sowohl Geschlecht als auch die Regionen als Kovariablen enthält, findet sich für Männer im 
- Tab. 1 Anzahl beobachteter und erwarteter Todesfälle im Untersuchungszeitraum (21.2. bis 21.3. 2020) in selektierten Gemeinden * aus 9 Regionen Italiens und in Summe $\left(\sum\right)$; SMR mit 95\%-Konfidenzintervall (95\%-KI).

\begin{tabular}{|c|c|c|c|c|c|}
\hline Region & Beobachtet & Erwartet & SMR & \multicolumn{2}{|c|}{ 95\%-KI } \\
\hline Piemonte e VdA & 1362 & 882 & 1,54 & 1,46 & 1,63 \\
\hline Lombardia & 10165 & 4768 & 2,13 & 2,09 & 2,17 \\
\hline Trentino-A.A. & 131 & 84 & 1,56 & 1,30 & 1,85 \\
\hline Veneto & 1208 & 839 & 1,44 & 1,36 & 1,52 \\
\hline Friuli-V.G. & 106 & 73 & 1,45 & 1,19 & 1,76 \\
\hline Liguria & 455 & 337 & 1,35 & 1,23 & 1,48 \\
\hline Emilia-Romagna & 2954 & 1687 & 1,75 & 1,69 & 1,82 \\
\hline Centro & 2012 & 1436 & 1,40 & 1,34 & 1,46 \\
\hline Sud e isole & 1475 & 1023 & 1,44 & 1,37 & 1,52 \\
\hline$\sum$ & 19868 & 11129 & 1,79 & 1,76 & 1,81 \\
\hline
\end{tabular}

> Tab. 2 Anzahl beobachteter und erwarteter Todesfälle unter Männern im Untersuchungszeitraum (21.2. bis 21.3. 2020) in selektierten Gemeinden * aus 9 Regionen Italiens und in Summe $\left(\sum\right)$; SMR mit 95\%-Konfidenzintervall (95\%-KI).

\begin{tabular}{|c|c|c|c|c|c|}
\hline Region & Beobachtet & Erwartet & SMR & \multicolumn{2}{|c|}{$95 \%-\mathrm{KI}$} \\
\hline Piemonte e VdA & 696 & 403 & 1,73 & 1,60 & 1,86 \\
\hline Lombardia & 5296 & 2205 & 2,40 & 2,34 & 2,47 \\
\hline Trentino-A.A. & 70 & 38 & 1,84 & 1,44 & 2,33 \\
\hline Veneto & 576 & 385 & 1,50 & 1,38 & 1,62 \\
\hline Friuli-V.G. & 51 & 34 & 1,50 & 1,12 & 1,97 \\
\hline Liguria & 213 & 152 & 1,40 & 1,22 & 1,60 \\
\hline Emilia-Romagna & 1517 & 780 & 1,95 & 1,85 & 2,05 \\
\hline Centro & 1000 & 683 & 1,46 & 1,37 & 1,56 \\
\hline Sud e isole & 740 & 515 & 1,44 & 1,34 & 1,54 \\
\hline$\sum$ & 10159 & 5195 & 1,96 & 1,92 & 1,99 \\
\hline
\end{tabular}

- Tab. 3 Anzahl beobachteter und erwarteter Todesfälle unter Frauen im Untersuchungszeitraum (21.2. bis 21.3. 2020) in selektierten Gemeinden * aus 9 Regionen Italiens und in Summe $\left(\sum\right)$; SMR mit 95\%-Konfidenzintervall (95\%- KI).

\begin{tabular}{|c|c|c|c|c|c|}
\hline Region & Beobachtet & Erwartet & SMR & \multicolumn{2}{|c|}{$95 \%-K I$} \\
\hline Piemonte e VdA & 663 & 477 & 1,39 & 1,29 & 1,50 \\
\hline Lombardia & 4868 & 2562 & 1,90 & 1,85 & 1,95 \\
\hline Trentino-A.A. & 60 & 45 & 1,33 & 1,02 & 1,72 \\
\hline Veneto & 630 & 453 & 1,39 & 1,28 & 1,5 \\
\hline Friuli-V.G. & 55 & 39 & 1,41 & 1,06 & 1,84 \\
\hline Liguria & 242 & 185 & 1,31 & 1,15 & 1,48 \\
\hline Emilia-Romagna & 1436 & 906 & 1,58 & 1,50 & 1,67 \\
\hline Centro & 1016 & 756 & 1,34 & 1,26 & 1,43 \\
\hline Sud e isole & 735 & 508 & 1,45 & 1,34 & 1,56 \\
\hline$\sum$ & 9705 & 5931 & 1,64 & 1,60 & 1,67 \\
\hline
\end{tabular}

* Gemeinden mit mehr als 20\% Zunahme an Todesfällen im Monat März 2020 im Vergleich zu den durchschnittlichen Todesfällen an denselben Tagen in den Jahren 2015-2019.

Vergleich zu Frauen eine um 20\% (95\%-KI 17\%-23\%) erhöhte SMR (mit Berücksichtigung einer Überdispersion: 16\%, 95\%-KI: 10\% bis $22 \%)$, gleichzeitig variiert die SMR zwischen den Regionen signifikant ( $p=0,006$, mit Berücksichtigung einer Überdispersion: $p=0,033)$. Diese Variation ist innerhalb der Männer stark ausgeprägt $(p<0,001)$, aber unter den Frauen nicht nachzuweisen $(p=0,995)$. 


\section{Diskussion}

Bereits zu Beginn des Auswertungs-Zeitfensters am 21. Februar gab es drastische Maßnahmen, um Gemeinden in Norditalien abzuriegeln. Am 4. März wurden auf Anordnung der Regierung Schulen und Universitäten in ganz Italien geschlossen. Am 8. März wurden die Lombardei und 14 weitere Provinzen zu einer ausgedehnten „Roten Zone“ oder Sperrzone erklärt, am 10.3.2020 ganz Italien ${ }^{5}$. Der hier untersuchte Zeitraum vom 21.02.2020 bis zum 21.3.2020 stellt somit nicht die Entwicklung der Mortalität bei unkontrolliertem Verlauf der Infektion mit SARS-Cov-2 dar, sondern beinhaltet auch Auswirkungen der ergriffenen Maßnahmen.

Bei der Mortalität zeigen sich erhebliche Unterschiede für den untersuchten Zeitraum 2020 im Vergleich zu den fünf früheren Jahren ( $\triangleright$ Tab. 1). Die Gesamt-SMR ist mit 1,8 fast verdoppelt. Die Vergleichszahl („Erwartet“) enthält auch die Wirkung der Grippewellen in den Vorjahren 2015 bis 2019.

Die SMR für Männer ( $>$ Tab. 2) liegt mit 1,96 (0,95-KI: 1,921,99) eindeutig höher als bei Frauen ( $>$ Tab. 3), 1,64 (0,95-KI: 1,601,67). Die Konfidenzbereiche überlappen sich nicht. Dieser Befund einer unter Männern höheren SMR ergibt sich nicht nur im Mittel, sondern in fast allen untersuchten Regionen. Das Poissonmodell mit Geschlecht und Regionen als Kovariablen bestätigt diesen Unterschied der Geschlechter im Zuwachs des relativen Sterberisikos als signifikant, auch nach Berücksichtigung einer möglichen Überdispersion.

Da die Todesfälle in [4] nicht nach Alter aufgeschlüsselt vorliegen, kann von uns nicht ermittelt werden, aus welchen Altersgruppen sich die erhöhte Mortalität ergibt.

In weiterem Datenmaterial des Nationalen Instituts für Statistik in Italien [5] stellt sich für den Zeitraum 1. März bis 21. März eine erhöhte Mortalität dar, die vom Norden nach Süden Italiens ab- und zwischen den Altersstrata 65-74 und über 75 Jahren zunimmt. EuroMOMO berichtet eine ausgeprägte Exzessmortalität in 2020 für Italien bis März ${ }^{6}$ und für die Gesamtheit aller teilnehmenden europäischen Länder bzw. Regionen Exzessmortalitäten für die Altersgruppe über 65 Jahre, aber auch für die Altersgruppe 15-64 Jahre?

Die großen Fallzahlen würden eine Differenzierung nach Todesursachen erlauben, z. B. nach Pneumonie, Herzinfarkten, Selbstmorden oder den in epidemiologischen Untersuchungen üblichen Gruppierungen von Endpunkten. Derartige Daten stehen bislang nicht zur Verfügung. Perspektivisch könnte eine solche Differenzierung Hypothesen und Hinweise ermöglichen, welche Mortalitätseffekte einer direkten Wirkung des Virus (z. B. Pneumonie) und welche ergriffenen Gegenmaßnahmen (z. B. Freitod infolge des physical-social distancing) zuzuordnen sein mögen.

Die Schätzungen der SMR variieren ausgeprägt zwischen den Regionen, von 1,35 in Liguria bis 2,13 in Lombardia (Homogenität wird im Poisson-Modell abgelehnt, auch bei Berücksichtigung einer Überdispersion). Diese Heterogenität zeigt sich nicht in beiden Ge-

5 lenca M \& Shaw D. The Slow Dragon and the Dim Sloth: What can the world learn from coronavirus responses in Italy and the UK? Posted on March 28, 2020. https://blogs.bmj.com/medical-ethics/2020/03/28/ the-slow-dragon-and-the-dim-sloth-what-can-the-world-learn-fromcoronavirus-responses-in-italy-and-the-uk/

6 https://www.euromomo.eu/graphs-and-maps: z-score

7 https://www.euromomo.eu/graphs-and-maps: number schlechtern, sondern stammt aus einer starken Variation der Schätzungen unter den Männern. Diese beobachtete Heterogenität ist u. a. durch eine unterschiedliche regionale Dynamik des Infektionsgeschehens begründbar. Die ausgewählten Regionen gelten als besonders betroffen und die so abgeleitete SMR überschätzt vermutlich den Befund bzw. die zu erwartenden Beobachtungen für Italien insgesamt.

Tatsächlich haben Colombo und Impicciatore [4] allein Gemeinden eingeschlossen, die im März 2020 mehr als 20\% überzählige Todesfälle hatten als im gleichen Zeitraum der Jahre 2015-2019. Üblicherweise würde dieser Ansatz zu der Frage nach den anderen Gemeinden führen. In der besonderen Corona-Situation kann die auf Gemeinden mit mehr als 20\%-Zunahmen fokussierte „Mortalitäts-Exzess-Lupe“ - bei Annahme sonst konstanter Determinanten für Todesfälle - aber ein Virus-Test-unabhängiges Instrument sein, tatsächliche Verbreitungen und vermutete Effekte des SARSCoV-2 zu suchen und zu beobachten. Mit diesen Annahmen könnte man das Zeitfenster der Untersuchung auch früher öffnen, um maskierte Mortalitätszunahmen bereits vor der ersten Meldung eines „COVID-19-Todesfalles“ in Italien zu finden. Indem man das Zeitfenster der Mortalitäts-Auswertungen länger offen hält oder später öffnet bzw. entsprechende Indikatorvariablen in Regressionsmodelle einsetzt, kann man den Verlauf von SARS-CoV-2-Effekten, aber auch von erwünschten und unerwünschten Wirkungen variabler Interventionen, überwachen und mit Mehrwert analysieren.

Die aktuelle „Lupe“ zeigt ausgeprägte Mortalitätsanstiege auch in Regionen Italiens, die nicht als Corona-Hotspots galten. Insofern können Mortalitätsdaten auch bei Fehlen repräsentativer Testergebnisse wichtige Hinweise auf die Verteilung oder Ausbreitung eines neu wirkenden Faktors - hier SARS-CoV-2 - sein. Zu Recht schreiben Colombo und Impicciatore [4]: „Die Feststellung der Anzahl der Todesfälle, die über dem Durchschnitt der letzten 5 Jahre liegen, ermöglicht nicht nur die Unterscheidung zwischen Todesfällen „durch“ und Todesfällen „mit“ Coronavirus zu überwinden, sondern auch die Größe der Dunkelziffer der Covid-19 zugeschriebenen Sterblichkeit zu schätzen“. Insofern können Mortalitätsdaten zu interessierenden Todesursachen und mit angemessener zeitlicher wie regionaler Auflösung wichtige epidemiologische Daten liefern und zu wesentlichen Erkenntnissen beitragen.

Der Erwartungswert der Anzahl an Todesfällen wird auf den Median gegründet. Hier wäre der Mittelwert geeigneter. Üblicherweise ist der Mittelwert bei rechtsschiefen Verteilungen größer als der Median, was bei Verwendung des Medians zu einer Effektüberschätzung führt. Eine Rechtsschiefe der Verteilung der Todeszahlen ist zu unterstellen, da die Anzahlen durch 0 nach unten beschränkt sind.

Es ist unklar, welchen Anteil die selektierten Gemeinden an der gesamten Population der Regionen ausmachen und wie sich die SMR-Ergebnisse aus diesen Gemeinden zu der für die jeweils gesamte Region ergebenden SMR verhalten. Weitergehende Schlussfolgerungen, z. B. zu relevanten lokalen Komponenten des epidemischen Verlaufs, evtl. als Folge von Wechselwirkungen mit Umweltbelastungen oder Versorgungsstrukturen, lassen sich somit nicht ableiten.

Diese Verzerrung durch die Selektion besonders betroffener Regionen und durch die Wahl des Medians als Vergleichspunkt wird 
in aktuellen Darstellungen zur Studie von Colombo und Impicciatore [4] nicht genügend beachtet ${ }^{8}$.

Grundsätzlich wird in diesen Analysen vorausgesetzt, dass sich Alters- und Geschlechtsverteilungen in den betrachteten Auswertegebieten nicht relevant zwischen dem Vergleichszeitraum 2015 bis 2019 und dem Expositionszeitraum 2020 unterscheiden. Diese Annahme erscheint unkritisch. SMR-Analysen gehen zudem davon aus, dass die Vergleichszahl („Erwartet“) sicher bestimmt ist, wodurch die Breiten der Konfidenzintervalle insbesondere bei kleineren Regionen unterschätzt sein könnten.

Eine Übertragbarkeit dieser ersten Ergebnisse auf Deutschland kann nicht begründet werden, auch nicht in die Zukunft Italiens. Im Anhang finden sich weitgehende Limitationen einer Anwendung der SMR-Befunde dieser Studie zur Schätzung der Krankheitslast („burden-of-disease“) durch SARS-CoV-2/COVID-19 in Italien.

Eine Einordnung der COVID-19 Pandemie im Vergleich zur schweren Influenza-Pandemie von 2017/2018 erscheint in Bezug auf publizierte deutsche [11, 12] und europäische [13] Analysen derzeit nicht möglich. Allerdings erlaubt das System EuroMOMO einen ersten groben Blick: Danach sind die bislang für die Gesamtheit der teilnehmenden europäischen Länder bzw. Regionen beobachteten Exzessmortalitäten im Jahr 2020 ähnlich zu den in 2016/2017 sowie 2017/2018 dokumentierten Exzessen ${ }^{9}$. Nota bene: Es bleibt künftigen Forschungsarbeiten vorbehalten, Antworten darauf zu geben, welche Bedeutung die ergriffenen massiven Maßnahmen für die derzeitigen Befunde haben.

Im Ausblick ist es dringend angezeigt, solche SMR-Analysen für Deutschland regelmäßig durchzuführen, differenziert nach Regionen, Geschlechtern, Altersgruppen und Todesursachen, um das Mortalitätsgeschehen in Folge der SARS-Cov-2-Pandemie belastbar zu beschreiben und im Verlauf auszuwerten. Tatsächlich können SMR-Auswertungen zur Früherkennung einer an Exzess-Todesfällen ablesbaren Pandemie und zur Überwachung der dynamischen Verbreitung eines Faktors wie SARS-CoV-2 beitragen. Darüber hinaus können sie genutzt werden, um sowohl erwünschte als auch unerwünschte Wirkungen (z. B. Freitod-assoziierte SMR) der ergriffenen Maßnahmen, die sich zeitlich und räumlich unterscheiden können, abzuschätzen und zu evaluieren.

In diesem Sinne weisen wir darauf hin, dass mit dem Masernschutzgesetz zum 1. November 2021 die gesetzliche Grundlage für eine bundesweite zeitnahe Mortalitäts-Surveillance ohnehin in Kraft treten wird [14]: „Dadurch kann zukünftig frühzeitig erkannt werden, ob im Falle einer Epidemie, während der Influenzasaison oder während einer Hitzewelle mehr Personen als im vergleichbaren Zeitraum der Vorjahre versterben. Diese Daten sind wichtig zur Einschätzung der Schwere der zuvor genannten Ereignisse, auf deren Grundlage ggf. Maßnahmen zur weiteren Eindämmung ergriffen werden können “. Auf europäischer Ebene gibt es das bereits genannte EuroMOMO ${ }^{10}$, in dem allerdings lediglich die Gesamtmortalität verfolgt wird und sich für Deutschland allein Hessen und Berlin beteiligen. Dies erlaubt keine Auswertung des todesursachenspezifischen Mortalitätsgeschehens in Deutschland.

8 https://www.tagesspiegel.de/politik/studie-zu-corona-sterblichkeit-initalien-forscher-gehen-von-doppelt-so-vielen-toten-aus/25711208.html

9 https://www.euromomo.eu/graphs-and-maps: number

10 http://www.euromomo.eu/
In der Gesamtschau bitten wir darum, dass die verantwortlichen Institutionen entsprechende epidemiologische Projekte zu den ohnehin regelmäßig in Deutschland erhobenen Mortalitätsdaten auflegen, um „Mortalitäts-Exzess-Lupen“ zu nutzen und Öffentlichkeit und Politik sachgemäß zu informieren.

\section{FAZIT FÜR DIE PRAXIS}

- Um das COVID-19 zuzuschreibende Mortalitätsgeschehen in Deutschland in 2020 belastbar zu beschreiben, sind Vergleiche mit Vorjahren notwendig.

- Beobachtete und erwartete Todesfälle, differenziert nach Regionen, Geschlechtern, Altersgruppen und Todesursachen sollten berichtet werden.

- Analysen von Standardisierten Mortalitäts-Ratios (SMRs) mit einer „Mortalitäts-Exzess-Lupe“ können zur Früherkennung einer an Todesfällen ablesbaren Pandemie und zur Überwachung der dynamischen Verbreitung eines Faktors wie SARS-CoV-2 beitragen.

- Berechnungen von SMRs und ihre Analysen mit geeigneten Regressionsmodellen werden auch empfohlen, um sowohl erwünschte als auch unerwünschte Wirkungen von ergriffenen Gegenmaßnahmen, die sich zeitlich und räumlich unterscheiden können, abzuschätzen und zu evaluieren.

\section{Danksagung}

Wir danken der Zeitschrift für den kompetenten Rapid ReviewProzess.

\section{Interessenkonflikt}

PM und TE geben keinen Interessenkonflikt an.

\section{Literatur}

[1] Onder G, Rezza G. Brusaferro S. Case-Fatality Rate and Characteristics of Patients Dying in Relation to COVID-19 in Italy. JAMA 2020; doi:10.1001/jama.2020.4683

[2] Grasseli G, Pesenti A, Cecconi M. Critical Care Utilization for the COVID-19 Outbreak in Lombardy, Italy. JAMA 2020; doi:10.1001/ jama.2020.4031

[3] an der Heiden M, Buchholz U. Modellierung von Beispielszenarien der SARS-CoV-2-Epidemie 2020 in Deutschland. Robert Koch-Institut. 2020; doi: $10.25646 / 6571.2$

[4] Colombo AD, Impicciatore R. La crescita della mortalità ai tempi del Covid-19. Analisi di 1.084 comuni italiani. I. stituto Carlo Cattaneo, Bologna. 2020. URL: https://www.cattaneo.org/2020/04/01/ gli-effetti-del-covid-19-sulla-mortalita/

[5] ISTAT. L'andamento dei decessi del 2020. Dati anticipatori sulla base del sistema ANPR, 31 marzo 2020. URL: https://www.istat.it/it/ files//2020/03/Decessi_2020_Nota.pdf

[6] Breslow NE, Day NE. Statistical methods in cancer research. Volume II - The design and analysis of cohort studies. Lyon: International Agency for Research on Cancer; 1987 
[7] Rothman KJ, Greenland S, Lash TL. Modern Epidemiology. 2. Auflage. Philadelphia: Lippincott, Williams and Wilkins; 2008

[8] Checkoway H, Pearce N, Kriebel D. Research methods in occupational epidemiology. $2^{\text {nd }}$ edition New York: Oxford University Press; 2004

[9] Cameron AC, Trivedi PK. Microeconometrics. Methods and Applications. New York: Cambridge University Press; 2005

[10] StataCorp. Stata Statistical Software: Release 14. Texas, USA: StataCorp LP, College Station; 2015

[11] Robert Koch-Institut. Bericht zur Epidemiologie der Influenza in Deutschland, Saison 2017/18, Berlin 2018. URL: https://influenza.rki. de/Saisonberichte/2017.pdf

[12] Robert Koch-Institut. Bericht zur Epidemiologie der Influenza in Deutschland, Saison 2018/19, Berlin 2019. URL: https://influenza.rki. de/Saisonberichte/2018.pdf

[13] Nielsen J et al. European all-cause excess and influenza-attributable mortality in the 2017/18 season: should the burden of influenza B be reconsidered? Clinical Microbiology and Infection, 2020. URL: https:// doi.org/10.1016/j.cmi.2019.02.011

[14] Küpke NK, Matysiak-Klose D, Siedler A et al. Gesetz für den Schutz vor Masern und zur Stärkung der Impfprävention (Masernschutzgesetz) Epid Bull 2020; 10: 3-5. doi 10.25646/6526

[15] Morfeld P, Erren TC. Warum ist die „Anzahl vorzeitiger Todesfälle durch Umweltexpositionen“ nicht angemessen quantifizierbar? Gesundheitswesen 2019; 81: 144-149

[16] Hammitt JK, Morfeld P, Tuomisto JT et al. Premature Deaths, Statistical Lives, and Years of Life Lost: Identification, Quantification, and Valuation of Mortality Risks. Risk Analysis 2020; 40: 674-695. doi 10.111/risa.13427

[17] Rabl A. Analysis of air pollution mortality in terms of life expectancy changes: relation between time series, intervention, and cohort studies. Environmental Health: A Global Access Science Source 2006; 5: 1. http://www.ehjournal.net/content/5/1/1

[18] Leksell L, Rabl A. Air pollution and mortality: quantification and valuation of years of life lost. Risk Analysis 2001; 21: 843-857

\section{Anhang}

Limitationen einer Anwendung der SMR-Befunde dieser Studie zur Schätzung der Krankheitslast („,burden-of-disease“) durch SARS-CoV- 2/COVID-19 in Italien.

NOTA BENE: Es bleibt künftigen Forschungsarbeiten vorbehalten, Antworten darauf zu geben, welche Bedeutung die ergriffenen massiven Maßnahmen für die derzeitigen Befunde haben.

Im Folgenden bieten wir eine spekulative Antwort auf die Frage, was aus den ermittelten SMR-Werten ableitbar ist, um die durch SARS-CoV-2/COVID-19 verursachte Krankheitslast der Bevölkerung („burden-of-disease“) in Form von „Anzahl verursachter Todesfälle“ oder „verursachte Verkürzung der Lebenserwartung“ zu quantifizieren.

Als grober Schätzwert für die Mortalität in modernen Industriegesellschaften kann $1 \%$ pro Jahr angesetzt werden. Unterstellen wir eine relative Erhöhung der Mortalität im betrachteten Monat unter Infizierten um $50 \%$ (die auf Basis des Medians der Todeszahlen der Vorjahre in selektierten Regionen berechnete Gesamt-SMR $=1,8$ ergäbe nominal $80 \%$ ) sowie eine Beschränkung dieses Mortalitätsexzesses auf 2 bis 3 Monate im Jahr ${ }^{11}$, so könnten wir zur groben Orientierung einen Faktor 5 zur Absenkung der im Monatszeitraum beobachteten Exzessmortalität auf das Jahresniveau ansetzen, d. h. die Mortalität wäre in Italien absolut um 0,1 \% pro Jahr in 2020 erhöht. Die grundsätzlich höhere Mortalität im Winter könnte einen niedrigeren Faktor als 5 begründen. Jedoch muss auch die zu erwartende Kompensation durch Todesfälle in Betracht gezogen werden, die sich ohne COVID-19 außerhalb des Zeitfensters der Untersuchung aber innerhalb des Jahres ereignet hätten (Harvesting). Diese beiden Effekte sind gegenläufig und werden wegen fehlender Quantifizierung im Folgenden vernachlässigt.

Es könnte naheliegend erscheinen, aus diesem groben Schätzwert (Mortalitätserhöhung um 0,1\% pro Jahr) die der Infektion mit SARS-Cov-2 zuzurechnenden Zahl der Verstorbenen wie folgt aus 60 Mio. Einwohnern Italiens zu berechnen: 0,1 \% x $60 \mathrm{Mio}=60000$ vorzeitige Todesfälle durch SARS-CoV-2 in Italien in 2020. Eine solche Rechnung ergibt aber nicht die durch das Virus verursachte Zahl der Todesfälle [„Anzahl verursachter Todesfälle“], selbst dann nicht, wenn die Erhöhung der Mortalität um 0,1\% exakt bekannt wäre [15]. Die Anzahl der betroffenen Todesfälle ist ohne Kenntnis des Wirkungsmechanismus nicht aus den zur Verfügung stehenden Mortalitätsdaten bestimmbar, und diese Zahl könnte tatsächlich wesentlich größer sein als eine solche Rechnung ergibt [16].

Aus denselben Gründen ist die Differenz zwischen 9705 beobachteten und 5931 erwarteten Todesfällen ( $\bullet$ Tab. 1) nicht die Anzahl der dem Virus zuzuschreibenden Todesfälle im Untersuchungszeitraum der selektierten Gemeinden in den neun Regionen, d. h.

11 Dies Zeitfenster von 2 bis 3 Monaten entspricht aktuellen Hochrechnungen zu COVID-19 (siehe Abb. 2a und 2b in https://www.dgepi.de/assets/ Stellungnahmen/Stellungnahme2020Corona_DGEpi-21032020.pdf) und Beobachtungen zu Influenza-Episoden der letzten Jahre (https://www. euromomo.eu/graphs-and-maps: number). Wir setzen den im Beobachtungsmonat ermittelten Mortalitätsexzess als repräsentativ für diesen Zeitraum an. 
dies ist nicht die Anzahl der durch den Virus dort verursachten, also zeitlich vorverlagerten Todesfälle $[15,16]$.

Aus der erhöhten Jahresmortalität lässt sich jedoch grundsätzlich die zusätzlich verlorene Lebenszeit bestimmen (siehe Appen$\operatorname{dix} A$ in [17]). Allerdings sollte hierfür die Altersverteilung des Mortalitätsexzesses bekannt sein. Da diese uns z.Zt. nicht zur Verfügung steht, kann lediglich der grobe Orientierungswert für die mittlere Änderung der Mortalitätsrate um 0,1 \% in Italien angesetzt werden. Bei einem Altersmedian der Bevölkerung Italiens von ca. 46 Jahren ${ }^{12}$ und einer zugehörigen Restlebenserwartung von ca. 38 Jahren ${ }^{13}$ ergibt sich eine grobe Schätzung der mittleren Änderung der Lebenserwartung unter den Einwohnern Italiens von $(1 / 1000) * 38$ Jahren = - 14 Tagen (siehe Gleichung 19 und Appendix B in [18]). Unter Annahme eines finalen Infektionsanteils von $60 \%$ der Quellpopulation (der Anteil hängt u. a. von der Effektivität des physical-social distancing ab, siehe auch die Hochrechnungen in [3]) ergäbe sich unter den Infizierten in Italien eine Änderung der Lebenserwartung um - 14 Tage/60 \% = - 23 Tage [,verursachte Verkürzung der Lebenserwartung“].

Wir betonen, dass diese Rechnungen spekulativ sind: Sie stützen sich auf Annahmen mit großen Unsicherheiten bzw. es fehlen wesentliche Daten (z. B. Altersverteilung der Exzessmortalität). Insofern dienen diese Rechnungen allein einer exemplarischen Illustration, was man mit welchen Informationen belastbar berechnen könnte und welche Limitationen die derzeit verfügbaren Informationen haben.

12 https://de.statista.com/statistik/daten/studie/248994/umfrage/ durchschnittsalter-der-bevoelkerung-in-den-eu-laendern/

13 https://de.wikipedia.org/wiki/Liste_von_L\%C3\%A4ndern_nach_ durchschnittlicher_Lebenserwartung Italien liegt in der Lebenserwartung bei Geburt um ca. 1,5 Jahre höher als Deutschland. Die Restlebenserwartung wird daher um 1 Jahr höher angesetzt als in Deutschland mit 37 Jahren. 